\title{
Estudio experimental del sistema radicular del pasto vetiver sometido a esfuerzos de tracción
}

\section{Experimental study of root system of vetiver grass subjected to tensile stress}

\author{
$\underline{\text { Jesús Alberto Torres }}^{(*)(* *)}$, Roberto José Torres ${ }^{(*)(* *)}$, Jackeline Coromoto Peña ${ }^{(*)}$, Ricardo Antonio Picón ${ }^{(*)(* * *)}$, Luiggi Méndez ${ }^{(* *)}$, \\ Ruben José Calderas $(* * * *)$
}

\section{RESUMEN}

Un estudio experimental del comportamiento mecánico del sistema radicular del pasto Vetiver se presenta en este trabajo. Se realizaron 305 ensayos de resistencia a la tracción al sistema radicular primario de la planta Vetiver, sembradas en diferentes suelos y estado de crecimiento. La metodología comienza desde la producción de la planta y sus cuidados en los diferentes tipos de suelos. Las raíces se prepararon para el ensayo según lo propone Mickovski y Van Beek (1). Los resultados obtenidos reflejan un incremento sustancial de su resistencia a la tracción en comparación con la tomada para los diseños de estabilización (75 MPa). Se establece que la carga máxima se incrementa según el diámetro de la raíz bajo una relación potencial, y el esfuerzo a tracción de la raíz de la Planta Vetiver alcanza una media y moda de 118.5 MPa y 131.71 MPa, respectivamente.

Palabras clave: Vetiveria zizanioides; Vetiver; Esfuerzos a tracción; Estabilización de talud; Ensayos experimental.

\section{ABSTRACT}

An experimental study of the mechanical behavior of the Vetiver grass root system is shown in this paper. Experimental tests of tensile strength (305) were carried out in the primary root system of the Vetiver plant, sown in different soils and different growth states. The methodology starts from the reproduction of the plant and its care in the different types of soil where they were planted. The method proposed by Mickovski and Van Beek was used to prepare experimental tests (1). The results obtained reflect a substantial increase in its tensile strength compared to the strength taken usually for the stabilization designs (75 MPa). The maximum load is increased according to the diameter of the root in a potential relationship, and the traction stress of the Vetiver plant root reaches a mean and moda of 118.5 MPa and 131.71 MPa, respectively.

Keywords: Vetiveria zizanioides; Vetiver tensile strength, slope stabilization, experimental test.

${ }^{*}$ ) Departamento de Obras Civiles y Geología. Universidad Católica de Temuco (UCT), Temuco (Chile).

(**) Departamento de Geomecánica. Universidad de Los Andes (ULA), Mérida (Venezuela).

(***) Lab. de Mecánica Estructural (LME). Universidad Centroccidental «Lisandro Alvarado» (UCLA), Barquisimeto (Venezuela).

(***) Programa de Ingeniería Civil. Universidad Pontificia Bolivariana (UPB), Montería (Colombia).

Persona de contacto/Corresponding author: jtorres@uct.cl (J. Torres)

ORCID: https://orcid.org/oooo-0002-3573-8350 (J. Torres); https://orcid.org/oooo-0oo2-3701-2561 (R. Torres);

https://orcid.org/oooo-0oo1-6025-6428 (J. Peña); https://orcid.org/oooo-ooo1-6356-7360 (R. Picón);

https://orcid.org/oooo-0oo3-1698-051X (L. Méndez); https://orcid.org/oooo-0002-9070-5084 (R. Calderas)

Cómo citar este artículo/Citation: Torres, Jesús A.; Torres, Roberto J.; Peña, Jackeline C.; Picón, Ricardo A.; Méndez, Luiggi; Calderas, Ruben J. (2020). Estudio experimental del sistema radicular del pasto vetiver sometido a esfuerzos de tracción. Informes de la Construcción, $72(560):$ e365. https://doi.org/10.3989/ic.70923

Copyright: (C) 2020 CSIC. Este es un artículo de acceso abierto distribuido bajo los términos de la licencia de uso y distribución Creative Commons Reconocimiento 4.0 Internacional (CC BY 4.0). 


\section{INTRODUCCIÓN}

La geotecnia, desde sus inicios, se ha preocupado por establecer las obras de ingeniería de forma segura sobre los suelos donde se emplazan, por ello se han generado distintos métodos de mejora de los mismos (2). En los últimos años, se han diseñado métodos de estabilización de taludes que tiendan a disminuir el impacto ambiental con la misma efectividad que los métodos tradicionales (3). En este sentido, la bioingeniería se presenta como una disciplina que busca obtener las ventajas de las plantas, mezclando en algunas oportunidades con elementos constructivos inertes, para proteger y estabilizar los suelos (4). Una de las técnicas más empleadas en la actualidad es la Tecnología del Pasto Vetiver (TPV), dadas las características físicas y mecánicas que exhibe su sistema radicular.

El Vetiver es una planta herbácea, gramínea, perenne, sin tallo aparente, forma macollas muy densas que van engordando y creciendo sin ser invasivas. No tiene, ni desarrolla rizomas o estolones, posee hojas largas, rígidas, de hasta 80 $\mathrm{cm}$ de largo y menos de $1 \mathrm{~cm}$ de ancho, muy resistentes y de bordes ásperos (5).

El Vetiver tiene una velocidad de crecimiento muy rápida, en seis meses la altura de la planta puede alcanzar dos metros. Las raíces crecen igual de rápido, alcanzando de tres a cuatro metros de profundidad en el primer año (5).

El sistema radicular primario es muy resistente, crece en dirección vertical, formando una barrera y un anclaje natural en el suelo, alcanzando hasta más de cinco metros de profundidad. Las raíces son rígidas, muy largas, verticales y de grosor uniforme. Se adaptan a todo tipo de terrenos y penetran incluso en las capas rocosas (5), (6). Son raíces muy fuertes que, en conjunto con su sistema secundario y terciario, forman una base esponjosa y muy ramificada que mantiene al suelo protegido y sujeto.

Existen numerosos estudios que describen la resistencia a la tracción de este sistema radicular. En ellas se concluye que la resistencia a la tracción será una función del diámetro y los coeficientes que controlan esta relación potencial dependerán de la especie (1), (7)-(11), estando determinado por la composición de la celulosa, proteína, pectina, entre otros factores (12). En general, se acepta el valor de $75 \mathrm{MPa}$ como resistencia a la tracción para la raíz en estudio, pese a que últimamente se han reportado valores superiores (10), (11). El valor mencionado corresponde a la resistencia del diámetro promedio.

La resistencia a la tracción de las fibras naturales en la bioingeniería es particularmente importante ya que el incremento en la resistencia de los suelos producto de la vegetación depende de la cantidad de biomasa presente y la resistencia a la tracción. Uno de los primeros modelos para predecir la rotura de suelos con raíces propone, a partir del criterio de Mohr-Coulomb, un incremento asociado a la resistencia a la tracción por unidad de área de suelo y la distorsión geométrica de la raíz (6), (13)-(15).

La resistencia que se moviliza por efecto de la raíz dependerá de la cantidad de elongación y la adherencia de la raíz en el suelo; así el efecto máximo solo puede ocurrir si las fibras se estiran lo suficiente y las raíces no pueden deslizarse ni extraerse del suelo («pull out»). Por esto, pueden presentar- se tres respuestas durante el corte de un suelo reforzado con raíces; el primero la rotura de las mismas, el segundo su elongación y, el tercero el deslizamiento de ellas (16).

Como se observa, en los últimos años existen intentos por establecer el comportamiento del sistema suelo raíz (6), (9), (13), (15), (17), sin embargo cuando se analizan los modelos de resistencia, existen propiedades de difícil determinación, como la adherencia, fricción suelo-raíz y el módulo de elasticidad de la raíz (especialmente en los modelos de estiramiento y deslizamiento).

Los modelos para obtener un factor de seguridad en taludes estabilizados con bioingeniería, asumen un sistema sueloraíz, homogéneo e isótropo, bajo el modelo de rotura de raíces, considerando un valor nominal de resistencia al material sin factor de reducción para el diseño (1), (6), (14), (16).

Por esta razón, y considerando las variaciones en los valores reportados hasta la fecha se realizaron ensayos experimentales para evaluar la resistencia a la tracción del sistema radicular primario del pasto vetiver, a diferentes edades y plantadas en diferentes suelos; que sirva de base para la generación de métodos de estabilización empleando la Tecnología de Pasto Vetiver, encontrándose en este estudio valores superiores a los reportados en la literatura.

\section{METODOLOGÍA}

La investigación se desarrolló bajo una secuencia metodológica cuyas etapas se describen a continuación:

\subsection{Caracterización de los suelos}

Como el objetivo final de la investigación es establecer la resistencia a la tracción de las raíces de pasto vetiver plantado en diferentes suelos y a diferentes estadios de crecimiento, se seleccionaron materiales de características diferentes para estudiar si el tipo de suelo afecta el crecimiento y madurez de las fibras naturales.

Se decide emplear una arcilla de baja compresibilidad (CL), una arena limosa (SM) y una arena uniforme con limos (SP-SM) según el Sistema Unificado de Clasificación de Suelos (SUCS).

Para la caracterización de las muestras se le realizan los ensayos granulométricos (18) y de límites de consistencia (19).

\subsection{Plantación y cuidado}

Se conoce que el sistema radicular primario del pasto vetiver tiene un crecimiento muy vertical, sin embargo para disminuir las perturbaciones geométricas de las mismas y controlar visualmente su crecimiento, se diseñaron una serie de recipientes de vidrio de $6 \mathrm{~mm}$ de espesor y un área cuadrada de $100 \mathrm{~cm}^{2}$, con alturas de 30 y $50 \mathrm{~cm}$.

Se planta la gramínea en los suelos previamente señalados, para ello se seleccionan esquejes sanos y bien desarrollados; una vez sembrados se les cortan las hojas para incrementar el desarrollo de sus raíces (17), como se observa en la Figura 1. Durante el período de crecimiento las plantas son monitoreadas constantemente y sometidas a las mismas condiciones de luz, humedad y temperatura, para disminuir las variables que pudieran afectar el crecimiento y madurez. 


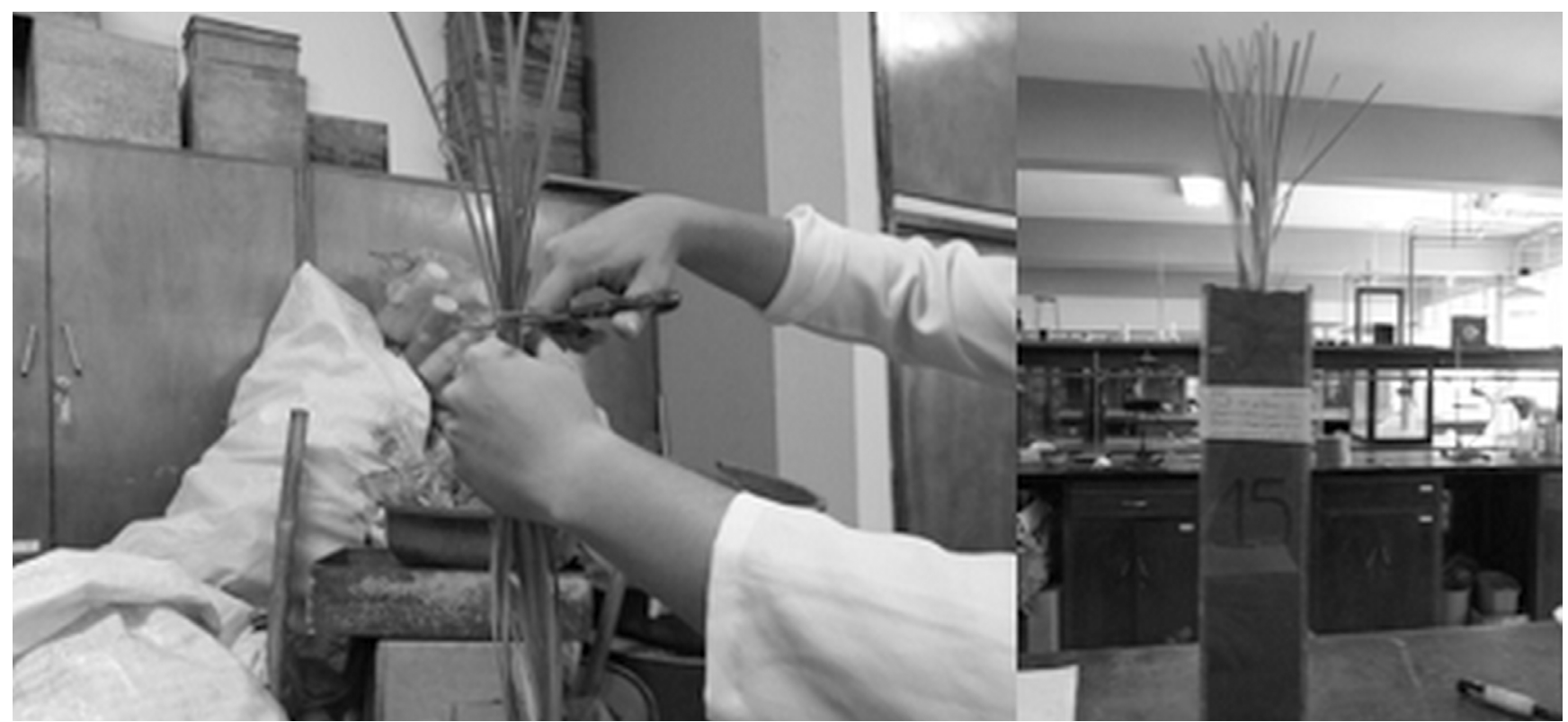

Figura 1. Recipientes y plantación del pasto vetiver.

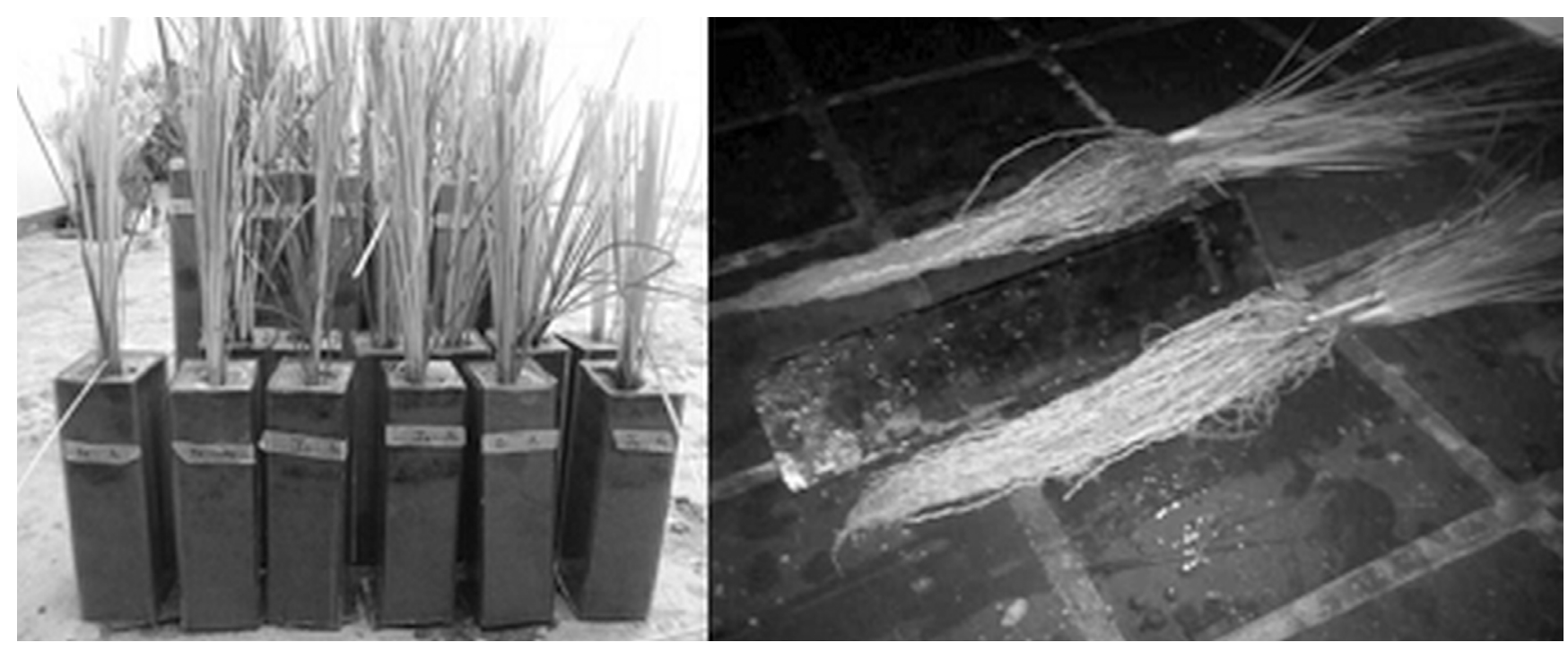

Figura 2. Preparación de las raíces para el ensayo. a) Recipientes previos al ensayo. b) raíces limpias.

\subsection{Ensayos de Resistencia a la tracción}

Transcurridos los tiempos establecidos $(4,6,8,16$ y 65 semanas) se preparan las raíces, para ello se desarman los recipientes de las plantas, se lavan cuidadosamente las raíces, evitando roturas, desgarres o cualquier perturbación sobre las fibras naturales (Figura 2) (1), (10).

En el ensayo, se recubren las mordazas sujetadoras con una película de corcho de $3 \mathrm{~mm}$ de espesor, para minimizar daños y cortes en la raíz, además de generar un mejor agarre (Figura 3). Se programa la máquina para un área transversal circular y a una velocidad de desplazamiento controlada. Se registran los diámetros y se llevan a la falla, el ensayo se considera válido si la raíz rompe en el tercio central de la longitud inicial (1).

La resistencia a la tracción se define como la carga última entre el área circular de la raíz no tensionada (Figura 4), tal como se muestra en la fórmula [1] (9), (13).

$$
\sigma_{t}=F_{\max } /\left(\left(\pi d^{2}\right) / 4\right)
$$

Donde $\mathrm{F}_{\max }$ es la máxima fuerza requerida para causar la rotura por tensión, d es el diámetro de la raíz no tensionada y $\sigma_{t}$ es la resistencia a la tracción.

\section{RESULTADOS}

\subsection{Caracterización de los suelos}

La selección de los suelos obedece al criterio de verificar si diversas condiciones granulométricas, proporciones y tipos de finos modifican el crecimiento y madurez de la planta. Adicionalmente, como se realiza un análisis temporal se busca establecer si existe variaciones de la resistencia en función de la edad de la planta.

En la Tabla 1 se muestran los resultados de los ensayos granulométricos, límites líquidos (LL), plásticos (LP) e ín- 

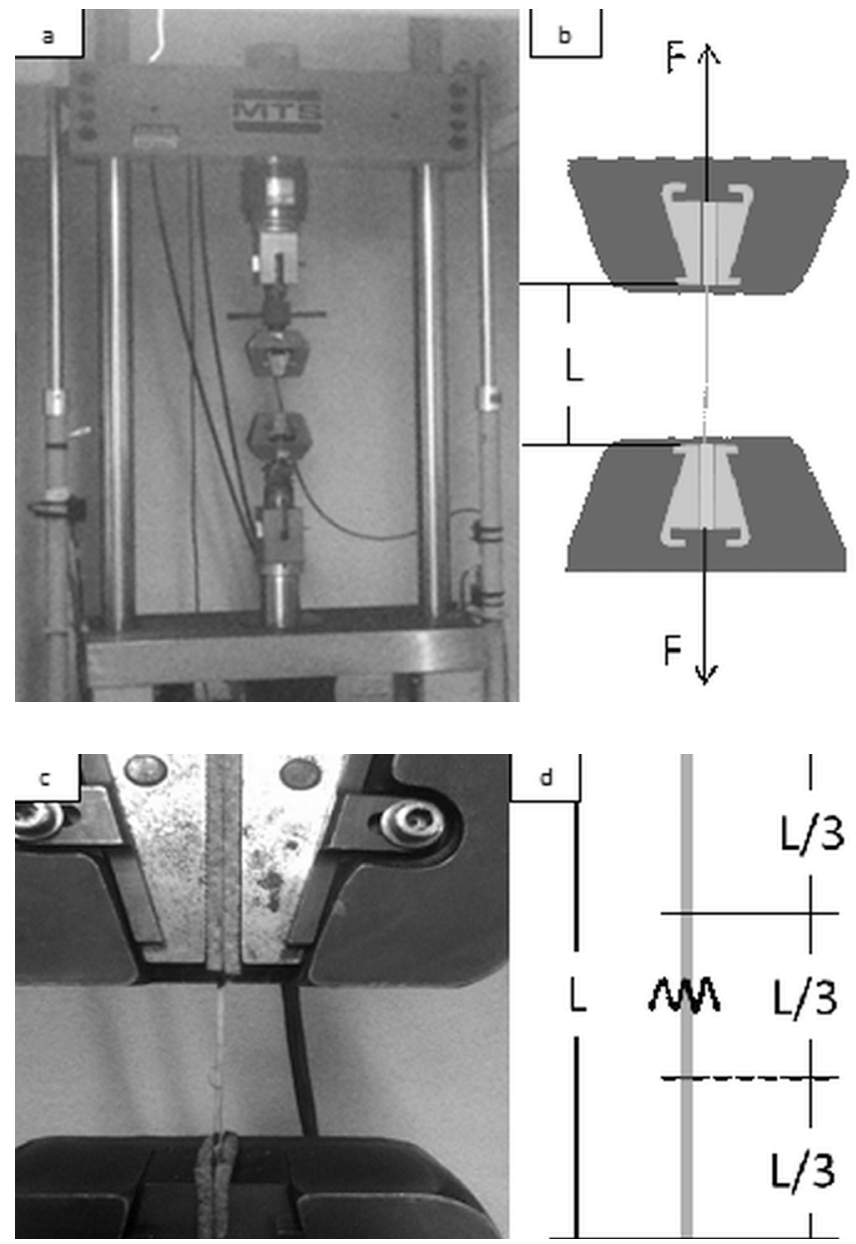

Figura 3. Diseño del ensayo de tracción. a) Máquina de tracción utilizada. b) Esquema del ensayo. c) Detalle de las mordazas sujetadoras y corcho para evitar la rotura de la raíz. d) Condición de rotura válida.

dices plásticos (IP), junto a la respectiva clasificación por el SUCS de las muestras seleccionadas para la plantación del vetiver.

Tabla 1. Clasificación de las muestras de suelo empleadas, según el SUCS.

\begin{tabular}{|l|l|c|l|c|}
\hline $\begin{array}{c}\text { Clasificación } \\
\text { SUCS }\end{array}$ & \multicolumn{2}{|l|}{ Granulometría (\%) } & \multicolumn{2}{c|}{$\begin{array}{c}\text { Límites de } \\
\text { Consistencia (\%) }\end{array}$} \\
\hline \multirow{3}{*}{ SM } & Gravas & 0.34 & LL & 21.84 \\
\cline { 2 - 5 } & Arenas & 73.85 & LP & NP \\
\cline { 2 - 5 } & Finos & 25.81 & IP & NP \\
\hline \multirow{3}{*}{ CL } & Gravas & 2.74 & LL & 30.35 \\
\cline { 2 - 5 } & Arenas & 12.74 & LP & 13.18 \\
\cline { 2 - 5 } & Finos & 84.52 & IP & 17.17 \\
\hline \multirow{3}{*}{ SP-SM } & Gravas & 0.00 & LL & 19.69 \\
\cline { 2 - 5 } & Arenas & 88.25 & LP & NP \\
\cline { 2 - 5 } & Finos & 11.75 & IP & NP \\
\hline
\end{tabular}

\subsection{Resistencia a la tracción de las raíces de Vetiver}

Se realizaron 305 ensayos válidos, correspondientes a un período de tiempo entre 4 y 65 semanas, en la Tabla 2 se muestra en detalle la distribución de los ensayos por tiempo y tipo de suelo. Dadas las características de la máquina de tracción

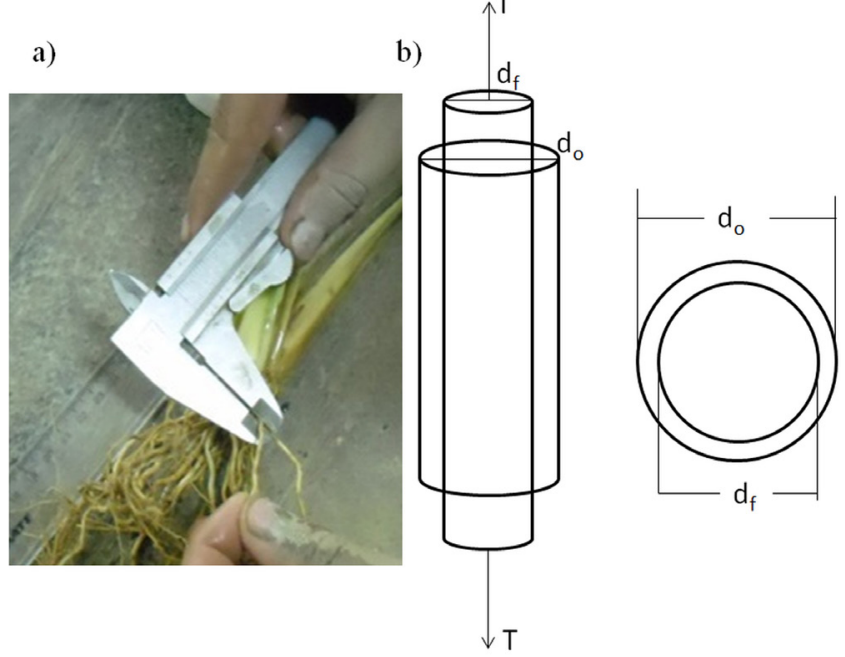

Figura 4. Diámetros tensionados y no tensionados. a) Medición del diámetro previo a la tensión (d ). b) Esquema de tracción directa y los diámetros asociados.

directa y lo pequeño de los diámetros se consideraron únicamente los diámetros mayores a $1 \mathrm{~mm}$.

Los resultados se presentan de manera individual, según el suelo de siembra y posteriormente el análisis conjunto.

Tabla 2. Ensayos válidos según tipo de suelo y tiempo de siembra.

\begin{tabular}{|l|c|c|c|}
\hline \multirow{2}{*}{ Tiempo de siembra } & \multicolumn{3}{|c|}{ Raíces ensayadas } \\
\cline { 2 - 4 } & SM & SP-SM & CL \\
\hline 4 Semanas & 14 & 13 & 0 \\
\hline 6 Semanas & 15 & 11 & 31 \\
\hline 8 Semanas & 30 & 24 & 0 \\
\hline 16 Semanas & 31 & 32 & 30 \\
\hline 65 Semanas & 0 & 0 & 74 \\
\hline Total & $\mathbf{9 0}$ & $\mathbf{8 0}$ & $\mathbf{1 3 5}$ \\
\hline
\end{tabular}

\subsubsection{Vetiver plantado en Arenas Limosas (SM)}

Se analizaron 90 roturas válidas, en ellas los diámetros de las raíces oscilaron entre $1.0 \mathrm{~mm}$ y $2.4 \mathrm{~mm}$, con una media de $1.56 \mathrm{~mm}$. La carga última tuvo un valor máximo de $392.4 \mathrm{~N}$, un mínimo de $91.23 \mathrm{~N}$, con una media de $226.43 \mathrm{~N}$ y una moda de 343.35 N (Tabla 3), al representar esta variable con los diámetros se aprecia que son proporcionales (Figura 5).

Tabla 3. Valores de diámetro no tensionado, carga última y resistencia a la tracción en arenas limosas (SM).

\begin{tabular}{|c|l|c|c|c|}
\hline \multicolumn{2}{|c|}{ Tipo de Suelo } & $\begin{array}{c}\text { Diámetro } \\
\text { (mm) }\end{array}$ & $\begin{array}{c}\text { Carga } \\
\text { última (N) }\end{array}$ & $\begin{array}{c}\text { Esfuerzo } \\
\text { (MPa) }\end{array}$ \\
\hline \multirow{4}{*}{} & Máximo & 2.40 & 392.40 & 168.45 \\
\cline { 2 - 5 } & Mínimo & 1.00 & 91.23 & 79.96 \\
\cline { 2 - 5 } & Media & 1.56 & 226.43 & 120.40 \\
\cline { 2 - 5 } SM & Moda & 1.90 & 343.35 & 144.37 \\
\cline { 2 - 5 } & Desv. Est. & 0.39 & 78.26 & 19.33 \\
\cline { 2 - 5 } & Curtosis & -1.19 & -1.25 & -0.50 \\
\cline { 2 - 5 } & Asimetría & 0.05 & 0.07 & 0.47 \\
\cline { 2 - 5 } & Coef. Variab. & 0.25 & 0.34 & 0.16 \\
\hline
\end{tabular}

Considerando la resistencia a la tracción se identificó que los valores máximos, mínimos y medios fueron 168.45 MPa, 


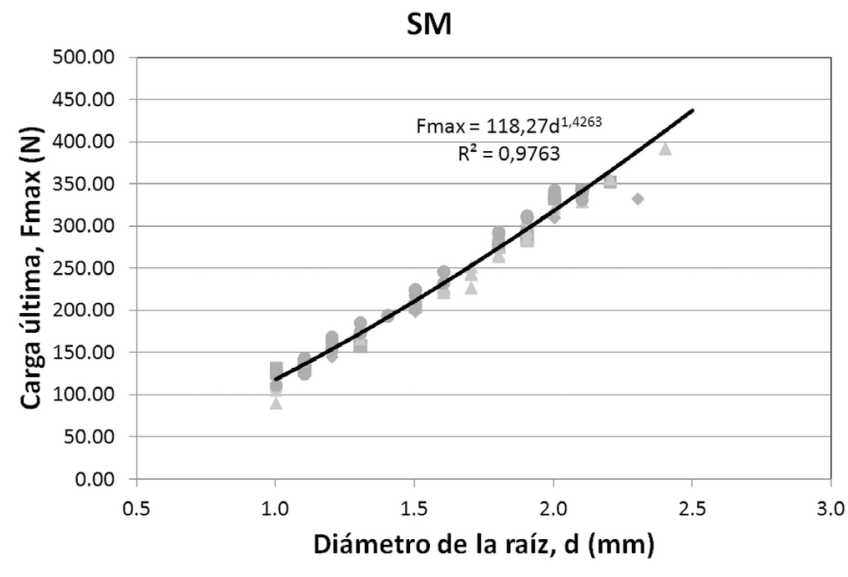

-4Semanas 6 Semanas $\triangle 8$ Semanas 16 Semanas -Tendencia

Figura 5. Carga última en función del diámetro para raíces de Vetiver plantadas en una arena limosa, ensayadas en diferentes estadios de crecimiento.

79.96 MPa y 120.40 MPa respectivamente, con una moda de 144.37 MPa. En este caso, la gráfica entre la variable considerada y los diámetros corresponde a una relación inversamente proporcional (Figura 6).

La relación entre la resistencia a la tracción y los diámetros, muestra lo descrito en investigaciones previas (1), (7), (8), (10), (11), una disminución de esfuerzo máximo a medida que se incrementa el diámetro, bajo una relación potencial. Las variables estadísticas, coeficiente de asimetría y curtosis (Tabla 3), permiten asumir una distribución de probabilidad normal, obteniéndose de esta manera que el $68.26 \%$ de los datos están comprendidos entre 101 y $139 \mathrm{MPa}$, valores correspondientes a la media más o menos una desviación estándar.

\subsubsection{Vetiver plantado en Arenas mal gradadas con limos (SP-SM)}

En este suelo, los diámetros estuvieron entre 1.0 y $2.2 \mathrm{~mm}$ con una media de $1.43 \mathrm{~mm}$ y una moda de $1.00 \mathrm{~mm}$. Los resultados son similares al caso anterior, cargas últimas que se incrementan conforme aumenta el diámetro de la raíz con un máximo, mínimo y media de 354.14 N, 99.08 N y $201.26 \mathrm{~N}$, respectivamente (Tabla 4 y Figura 7).

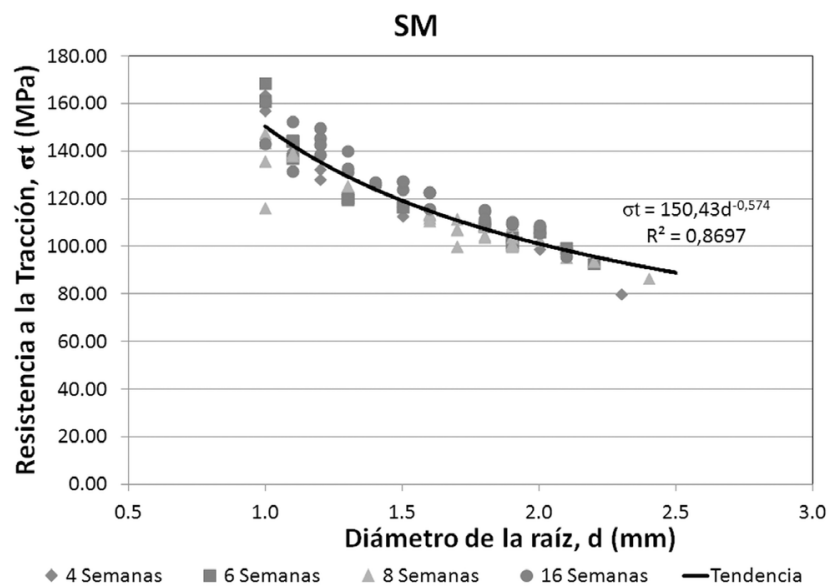

Figura 6. Resistencia a la tracción en función del diámetro para raíces de Vetiver plantadas en una arena limosa, ensayadas en diferentes estadios de crecimiento.
Tabla 4. Valores de diámetro no tensionado, carga última y resistencia a la tracción en arenas mal gradadas con limos (SP-SM).

\begin{tabular}{|c|l|c|c|c|}
\hline \multicolumn{2}{|c|}{ Tipo de Suelo } & $\begin{array}{c}\text { Diáametro } \\
(\mathbf{m m})\end{array}$ & $\begin{array}{c}\text { Carga } \\
\text { última (N) }\end{array}$ & $\begin{array}{c}\text { Esfuerzo } \\
(\mathbf{M P a})\end{array}$ \\
\hline \multirow{7}{*}{ SP-SM } & Máximo & 2.20 & 354.14 & 163.46 \\
\cline { 2 - 5 } & Mínimo & 1.00 & 99.08 & 91.26 \\
\cline { 2 - 5 } & Media & 1.43 & 201.26 & 125.69 \\
\cline { 2 - 5 } & Moda & 1.00 & 149.11 & 131.71 \\
\cline { 2 - 5 } & Desv. Est. & 0.37 & 75.03 & 18.98 \\
\cline { 2 - 5 } & Curtosis & -1.06 & -0.93 & -1.00 \\
\cline { 2 - 5 } & Asimetría & 0.48 & 0.58 & 0.13 \\
\cline { 2 - 5 } & Coef. Variab. & 0.26 & 0.37 & 0.15 \\
\hline
\end{tabular}

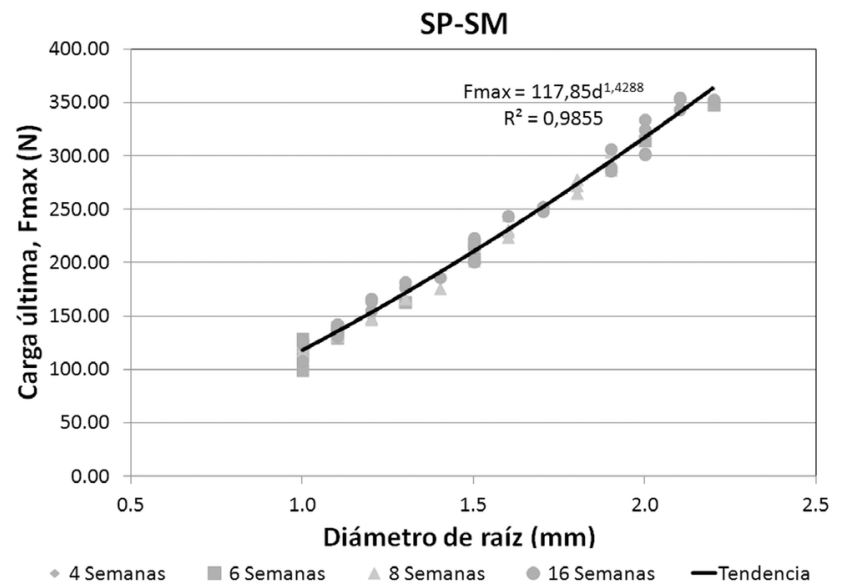

Figura 7. Carga última en función del diámetro para raíces de Vetiver plantadas en una arena mal gradada con limos, ensayadas en diferentes estadios de crecimiento.

La resistencia a la tracción, conserva el comportamiento descrito para las arenas limosas, disminuyendo según aumenta el diámetro de la raíz, siendo el registro más elevado 163.46 $\mathrm{MPa}$, el mínimo 91.26 MPa y la media 125.69 MPa, con una moda de 131.71 MPa (Tabla 4 y Figura 8).

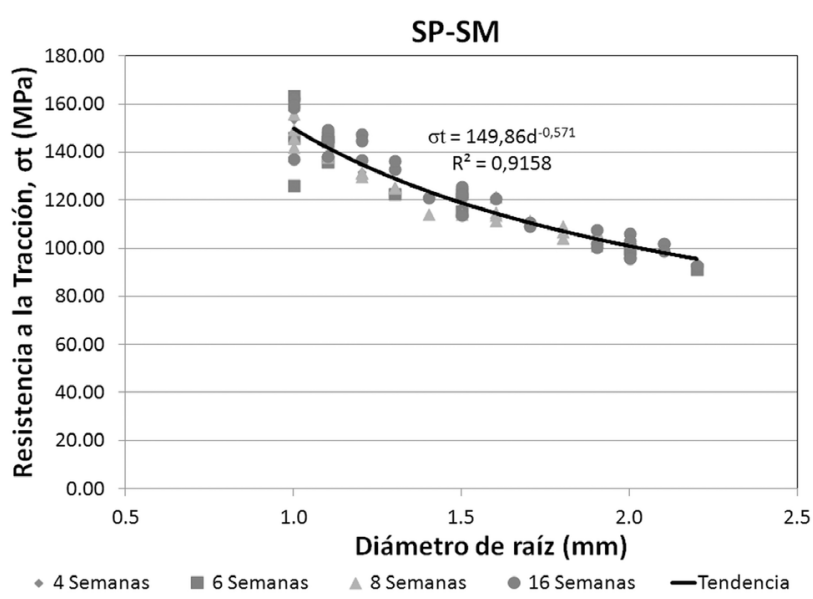

Figura 8. Resistencia a la tracción en función del diámetro para raíces de Vetiver plantadas en una arena mal gradada con limos, ensayadas en diferentes estadios de crecimiento.

3.2.3. Vetiver plantado en Arcillas de baja compresibilidad (CL)

Los diámetros para los ensayos válidos oscilaron entre 1.0 mm y $3.3 \mathrm{~mm}$, con una media de $1.80 \mathrm{~mm}$. Los resultados de 
estos ensayos muestran las tendencias previamente señaladas, la carga última varió entre 104.97 N y $638.63 \mathrm{~N}$, con un valor medio de $274.04 \mathrm{~N}$ y una moda de $149.11 \mathrm{~N}$ (Tabla $5 \mathrm{y}$ Figura 9); por su parte la resistencia a la tracción fluctuó entre $74.59 \mathrm{MPa}$ y $170.95 \mathrm{MPa}$, con una media y moda de 112.96 y 131.79 MPa respectivamente (Tabla 5 y Figura 10).

Los datos y análisis estadísticos señalan el 68.26\% de las resistencias máximas se encuentran entre 91.18 y $134.74 \mathrm{MPa}$, bajo el principio de ajuste a una distribución normal.

Tabla 5. Valores de diámetro no tensionado, carga última y resistencia a la tracción en una arcilla de baja compresibilidad.

\begin{tabular}{|l|l|c|c|c|}
\hline \multicolumn{2}{|c|}{ Tipo de Suelo } & $\begin{array}{c}\text { Diámetro } \\
\text { (mm) }\end{array}$ & $\begin{array}{c}\text { Carga } \\
\text { última (N) }\end{array}$ & $\begin{array}{c}\text { Esfuerzo } \\
(\text { MPa) }\end{array}$ \\
\hline \multirow{4}{*}{} & Máximo & 3.30 & 638.63 & 170.95 \\
\cline { 2 - 5 } & Mínimo & 1.00 & 104.97 & 74.59 \\
\cline { 2 - 5 } CL & 1.77 & 274.04 & 112.96 \\
\cline { 2 - 5 } & Modia & 1.50 & 149.11 & 131.71 \\
\cline { 2 - 5 } & Desv. Est. & 0.57 & 125.71 & 21.78 \\
\cline { 2 - 5 } & Curtosis & 0.06 & 0.47 & -0.07 \\
\cline { 2 - 5 } & Asimetría & 0.77 & 0.96 & 0.41 \\
\cline { 2 - 5 } & Coef. Variab. & 0.32 & 0.45 & 0.19 \\
\hline
\end{tabular}

$\mathrm{CL}$

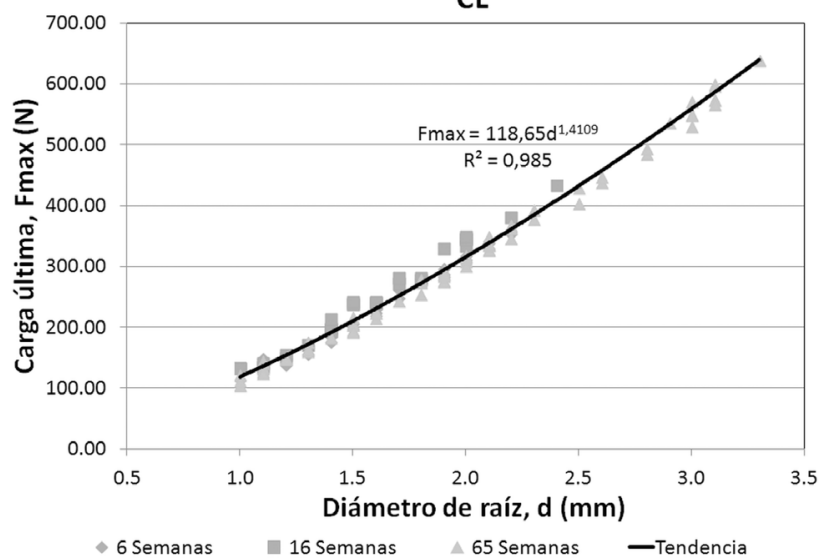

Figura 9. Carga última en función del diámetro para raíces de Vetiver plantadas en una arcilla de baja compresibilidad, ensayadas en diferentes estadios de crecimiento.

$\mathrm{CL}$

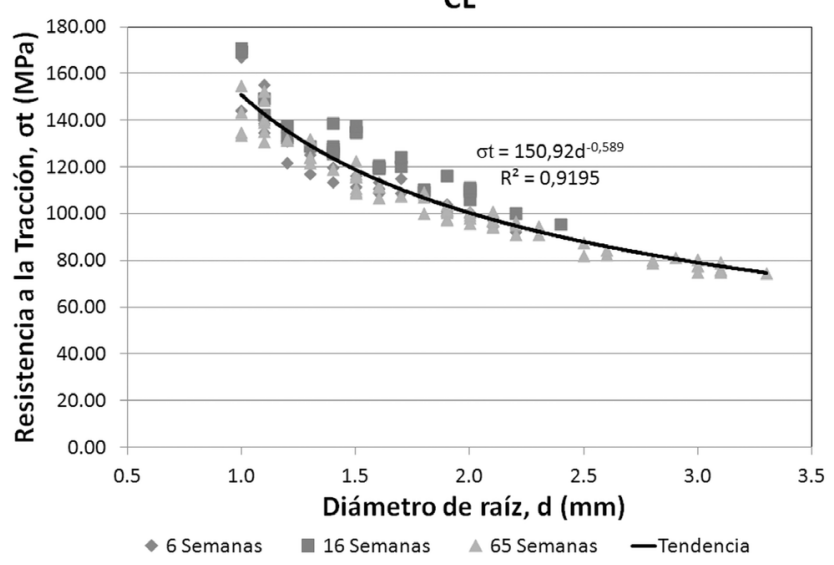

Figura 10. Resistencia a la tracción en función del diámetro para raíces de Vetiver plantadas en una arcilla

de baja compresibilidad, ensayadas en diferentes estadios de crecimiento.

\subsubsection{Análisis Integrado}

Como se observa en los apartados anteriores, las tendencias son similares en todos los suelos considerados, sin embargo las ecuaciones de las curvas de ajuste presentan ligeras variaciones en los coeficientes y exponentes, lo cual generó la inquietud si la madurez de la raíz y su consecuente resistencia, dependía directamente del tipo de suelo en el cual estuviera sembrado.

En la Tabla 6 y Figura 11 se observan, la carga última y diámetros de manera general, sin diferenciar edades ni tipo de suelo.

Tabla 6. Datos estadísticos de resistencia a la tracción carga última y diámetro no tensionado de la raíz para todos los tipos de suelo y edades.

\begin{tabular}{|c|l|c|c|c|}
\hline \multicolumn{2}{|c|}{ Tipo de Suelo } & $\begin{array}{c}\text { Diámetro } \\
(\mathbf{m m})\end{array}$ & $\begin{array}{c}\text { Carga } \\
\text { última (N) }\end{array}$ & $\begin{array}{c}\text { Esfuerzo } \\
(\text { MPa) }\end{array}$ \\
\hline \multirow{7}{*}{ General } & Máximo & 3.30 & 638.63 & 170.95 \\
\cline { 2 - 5 } & Mínimo & 1.00 & 91.23 & 74.59 \\
\cline { 2 - 5 } & Media & 1.62 & 240.90 & 118.50 \\
\cline { 2 - 5 } & Moda & 1.10 & 149.11 & 131.71 \\
\cline { 2 - 5 } & Desv. Est. & 0.49 & 105.76 & 20.99 \\
\cline { 2 - 5 } & Curtosis & 0.81 & 1.64 & -0.43 \\
\cline { 2 - 5 } & Asimetría & 0.89 & 1.15 & 0.24 \\
\cline { 2 - 5 } & Coef. Variab. & 0.31 & 0.44 & 0.18 \\
\hline
\end{tabular}

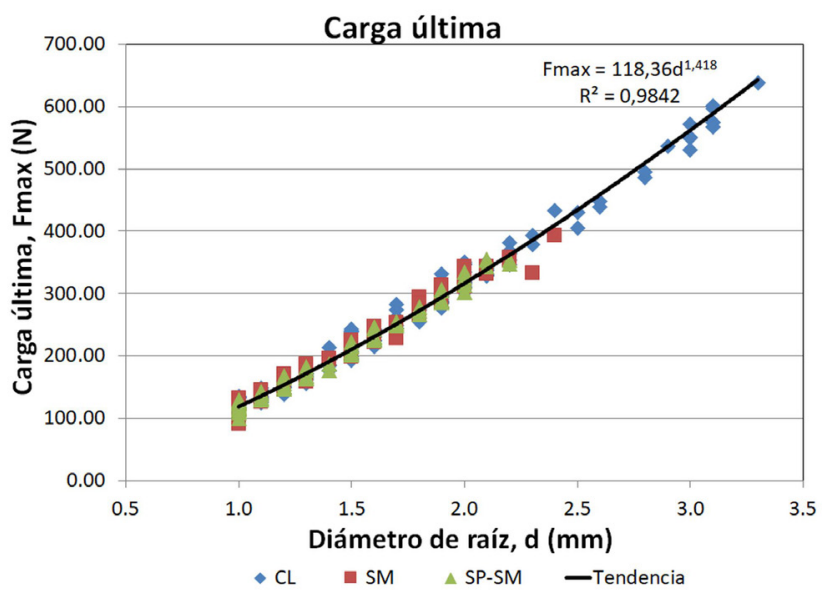

Figura 11. Carga última en función del diámetro para raíces de Vetiver plantadas en diversos tipos de suelos, ensayadas en diferentes estadios de crecimiento.

Bajo este análisis se establece que la carga última a la tracción tiene un comportamiento potencial, dado por la ecuación [2].

$$
F_{\max } \approx 118 d^{1.418}
$$

Donde, $\mathrm{F}_{\max }$ es la carga última a la tracción soportada en Newton y d es el diámetro de la raíz no tensionada en milímetros.

La resistencia a la tracción, cuando se correlaciona con el diámetro (Tabla 6 y Figura 12), exhibe una tendencia a la disminución, según una relación potencial, coincidiendo con lo reportado previamente (1), (7), (8), (10), (11), tal como se establece en la ecuación [3].

$$
\sigma_{t} \approx 150 d^{-0.582}
$$




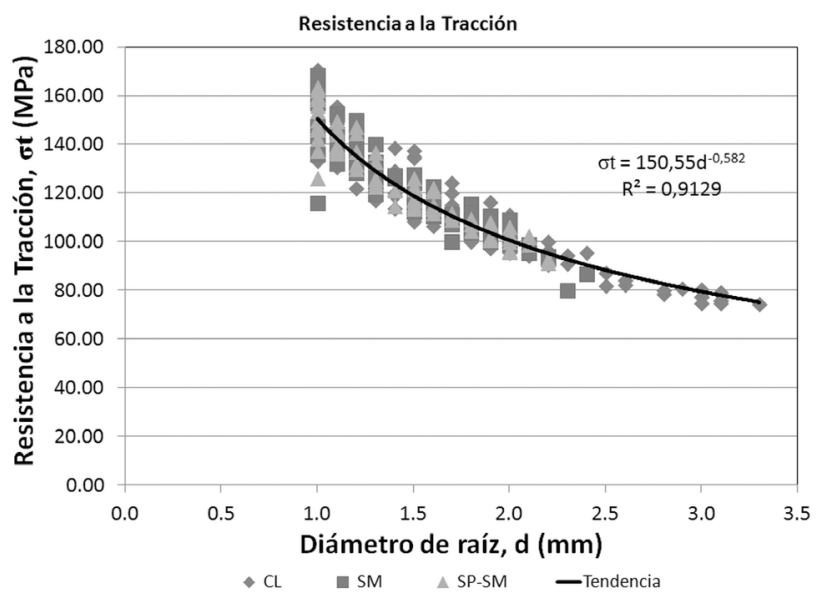

Figura 12. Resistencia a la tracción en función del diámetro para raíces de Vetiver plantadas en diversos tipos de suelos, ensayadas en diferentes estadios de crecimiento.

Donde, $\sigma_{t}$ es la resistencia a la tracción en MPa y d es el diámetro de la raíz no tensionada en milímetros.

La distribución experimental de la resistencia puede ajustarse a un comportamiento normal (Tabla 6 y Figura 13). En ella se destacan varios elementos importantes, en primer lugar que el valor del percentil 50 en la distribución experimental corresponde a $115.84 \mathrm{MPa}$, mientras que la media teórica es 118.50 MPa, lo que representa una diferencia cercana al $2 \%$.

Adicionalmente, se destaca que el 95\% de los datos experimentales obtenidos en toda la muestra se concentra en el rango comprendido entre 76.5 y 160.5 MPa. En general, se observa que los valores de resistencia obtenidos son superiores a los reportados por distintos autores (1), (9), (13), (17), pero similares a los indicados por Méndez, et al (10), destacando que en este caso se procesaron un número significativamente mayor de muestras y plantadas en diferentes tipos de suelos.

Se debe considerar que lo reportado en esta investigación es para condiciones de climas tropicales, bajo condiciones de luz y humedad controladas, sin someter a estrés hídrico a la planta, lo cual puede incidir en el crecimiento y desarrollo de las raíces. Se enfatiza que el valor de diseño de $75 \mathrm{MPa}$ indicado por los antecedentes proviene del ajuste potencial propuesto en base al diámetro medio de sus investigaciones, sin embargo, los diámetros registrados en este trabajo difieren significativamente de los reportados.

Se realizó un análisis de varianza (ANOVA) para identificar la relación entre los tipos de suelo y edad de siembra de la planta con la resistencia a la tracción. En primera instancia, se relacionó esta última variable con los tipos de suelo y considerando los valores de F y F crítico, 10.36 y 3.03 respectivamente, existe una relación estadísticamente significativa. Este mismo análisis se realizó para las edades y en función del $\mathrm{F}=13.48$ y Fcrítico=2.40, se concluye que también existe una relación estadísticamente significativa.

El ANOVA para dos factores (tipo de suelo y edad) se efectuó para las semanas 6 y 16, ya que se disponía igual número de muestras para los suelos en estudio; el resultado para la interacción de los factores $\left(\mathrm{F}=0.30, \mathrm{~F}_{\text {crítico }}=3.15\right)$ indica que no existe una relación significativa, esto permite concluir que se deben considerar otros modelos para establecer la relación (20).

\section{CONCLUSIONES}

La resistencia a la tracción de las raíces de vetiver, obtenida para todos los tipos de suelo seleccionados, supera sustancialmente lo reportado en las diversas referencias asociadas al tema. En este caso se obtienen valores que varían entre 74.59 MPa y 170.95 MPa, con una media de 118.50 MPa, una moda de 131.71 MPa y una desviación estándar de 20.99 MPa, lo cual representa un incremento significativo respecto a lo reportado por otros autores. La distribución experimental de la resistencia a la tracción puede ajustarse a un comportamiento normal. Bajo esta hipótesis, el valor nominal de 75 $\mathrm{MPa}$, que se emplea habitualmente, corresponde a un fractil del $5 \%$, aproximadamente.

La carga última varía en función del diámetro, fluctuando entre $91.23 \mathrm{~N}$ y $638.63 \mathrm{~N}$, con un promedio de $240.90 \mathrm{~N}$, una moda de $149.11 \mathrm{~N}$ y una desviación de $105.76 \mathrm{~N}$. Se propone una variación de la carga última en función del diámetro de forma potencial (Figura 10, Ecuación [2]).

Las tendencias registradas coinciden en buena medida con las descritas previamente, considerando que los coeficientes de correlación obtenidos son mayores a los reportados, así
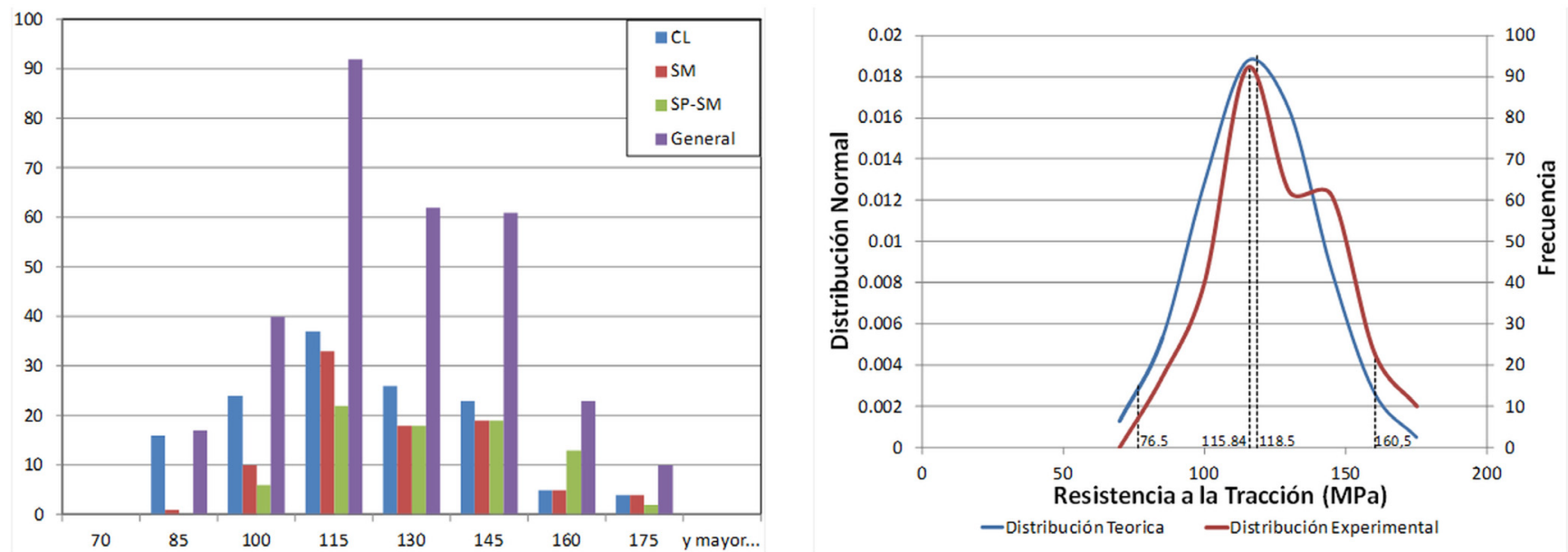

Figura 13. Histograma de Frecuencia de la resistencia a la tracción para raíces de Vetiver plantadas en diversos tipos de suelos, ensayadas en diferentes estadios de crecimiento. 
como un muestreo superior. Se destacan las condiciones de crecimiento y desarrollo de las plantas ya que el contenido y tipo de celulosa, entre otros factores, pueden influir significativamente en el comportamiento mecánico de la raíz.

Las técnicas utilizadas para analizar la influencia de la edad y tipo de suelo sobre la resistencia a la tracción indican que existen relaciones estadísticamente significativas. Sin embargo, para establecer la relación entre las variables se requieren emplear modelos más complejos.

Se recomienda realizar estudios de tracción y mediciones de contenido y tipo de celulosa sobre la raíz para analizar el efec- to de estas variables sobre la resistencia; así como estudios de extracción de plantas en suelo para establecer el efecto de «pull out» y su influencia en la estabilidad de un suelo reforzado con raíces.

\section{AGRADECIMIENTOS}

Agradecemos la colaboración y el apoyo del personal adscrito a los laboratorios de Mecánica de Suelos y Pavimentos, de Materiales y Ensayos, y de Análisis Experimental de Esfuerzos de la Universidad de los Andes (Venezuela) en la realización de todos los ensayos de la presente investigación.

\section{REFERENCIAS}

(1) Mickovski, S. B. and van Beek, L. P. H., (2009), "Root morphology and effects on soil reinforcement and slope stability of young vetiver (Vetiveria zizanioides) plants grown in semi-arid climate”, Plant Soil, vol. 324, no. 1-2, pp. 43-56.

(2) López Ruiz, A. and Ruiz, A. L., (1983), «Mejora geotécnica del suelo», Inf. la Construcción, vol. 35, no. 354, pp. 37-49. https://doi.org/10.3989/ic.1983.v35.i354.2001

(3) Claver Parias, I., Díaz Martín, M., and Martín, M. D., (1993), «Proyecto de restauración de los taludes y zonas anejas a una autovía», Inf. la Construcción, vol. 45, no. 425-426, pp. 123-134. https://doi.org/10.3989/ic.1993.v45.1425426.1189

(4) García-Vega, A., Sanz-Ronda, F. J., Fuentes-Pérez, J. F., Navarro-Hevia, J., Martínez-Rodríguez, A., and Martínez-Rodríguez, A., (2014), «Bases metodológicas para el cálculo de muros entramados de madera con vegetación o muros Krainer», Inf. la Construcción, vol. 66, no. 533, p. e012. https://doi.org/10.3989/ic.12.072

(5) Smyle, J., Taller de bioingenieria para la construccion post mitch; experiencia con el uso de Vetiver para la proteccion y estabilizacion de infraestructura, memoria. BANCO MUNDIAL, 1999.

(6) Wu, T. H., McOmber, R. M., Erb, R. T., and Beal, P. E., (1988), "Study of Soil-Root Interaction”, J. Geotech. Eng., vol. 114, no. 12, pp. 1351-1375.

(7) Bischetti, G. B. et al., (2005), "Root Strength and Root Area Ratio of Forest Species in Lombardy (Northern Italy)", Plant Soil, vol. 278, no. 1-2, pp. 11-22. https://doi.org/10.1007/s11104-005-0605-4

(8) Baets, S. De, Poesen, J., Reubens, B., Wemans, K., Baerdemaeker, J. De, and Muys, B., "Root tensile strength and root distribution of typical Mediterranean plant species and their contribution to soil shear strength", Plant and Soil, vol. 305. Springer, pp. 207-226. https://doi.org/10.1007/s11104-008-9553-0

(9) Nilaweera, N. and Grass, D. H., "Assessment of strength properties of vetiver grass roots in relation to slope stabilization”, in In Vetiver: A Miracle Grass, 1996.

(10) Méndez, L., Rojas, W., Torres, J., Torres, R., Rada, M., and Calderas, R., (2014), «Resistencia a la traccion del sistema radicular del vetiver (vetiveria zizanioides\} plantado en un suelo granular», Geominas, vol. 42, no. 64, pp. 165-170.

(11) Noorasyikin, M. N. and Zainab, M., (2016), "A Tensile Strength of Bermuda Grass and Vetiver Grass in Terms of Root Reinforcement Ability Toward Soil Slope Stabilization”, IOP Conf. Ser. Mater. Sci. Eng., vol. 136, no. 1, p. 012029. https://doi.org/10.1088/1757-899X/136/1/012029

(12) $\mathrm{Hu}$, X. et al., (2013), "An exploratory analysis of vegetation strategies to reduce shallow landslide activity on loess hillslopes, Northeast Qinghai-Tibet Plateau, China”, J. Mt. Sci., vol. 10, no. 4, pp. 668-686. https://doi.org/10.1007/s11629013-2584-x

(13) Wu, T. H., "Slope stabilization", in Slope stabilization and erosion control a bioengineering approach, R. P. C. (Royston P. C. Morgan and R. J. (R. J. Rickson, Eds. London: E \& FN Spon, 1995, pp. 233-265.

(14) L.J., W., (1977), "The shear resistance of root-permeated homogeneous and stratified soil”, J. Soil Sci. Soc. Am.

(15) Styczen, M. E. and Morgan, R. P. C., "Engineering properties of vegetation”, R. P. C. (Royston P. C. Morgan and R. J. (R. J. Rickson, Eds. London: E \& FN Spon, 1995, p. 57.

(16) Gray, D. H. and Barker, D., "Root-soil mechanics and interactions", American Geophysical Union (AGU), 2004, pp. 113123.

(17) Paul Truong, T. T. V. and E. P., Vetiver System Applications Technical Reference Manual, Second Edi., no. July. North Charleston SC, United States: Createspace Independent Publishing Platform, 2008.

(18) “ASTM D422 - 63(2007)e2 Standard Test Method for Particle-Size Analysis of Soils (Withdrawn 2016)", International, West Conshohocken, 2007. [Online]. Available: https://www.astm.org/Standards/D422. [Accessed: 30-Oct-2019].

(19) “ASTM D4318 : Standard Test Methods for Liquid Limit, Plastic Limit, and Plasticity Index of Soils”, International, 2017. [Online]. Available: https://global.ihs.com/doc_detail.cfm?document_name=\&item_s_key=00018514\&item_ key_date $=810831$. [Accessed: 30-Oct-2019].

(20) Barreto, W., Torres, J., Torres, R., Gonzalez, L., and Picón, R., (2019), "Modelling Tensile Strength of Vetiver Roots Using Artificial Neural Networks”, Rev. Tec. la Fac. Ing. Univ. del Zulia, vol. Especial, pp. 154-159. https://doi.org/10.22209/ rt.ve2019ao1 